\title{
Fat microstructure of yogurt as assessed by x-ray microtomography
}

\author{
J. Laverse, ${ }^{\star}$ M. Mastromatteo, † P. Frisullo, ${ }^{\star}$ M. Albenzio, ${ }^{\star} \ddagger$ D. Gammariello, $\neq$ and M. A. Del Nobile ${ }^{\star}{ }^{1}$ \\ *Department of Food Science, \\ †Department of Science of Production and Innovation in Agro-food Mediterranean Systems, and \\ ‡Institute for Research and Biotechnological Applications for Security and Valorization of Local Products and Qualità, University of Foggia, \\ via Napoli, 25 - 71100 Foggia, Italy
}

\begin{abstract}
In this work, the x-ray microtomography $(\mu \mathrm{CT})$ technique was used for the analysis of fat microstructure and quantification of fat in 7 types of yogurts. The dynamic mechanical properties of the yogurt samples also were studied using a controlled-strain rotational rheometer and correlated to the fat microstructure. Five types of commercially produced and 2 homemade yogurts, chosen to exhibit variability in terms of visible structure of fat, were used for this experiment. Appropriate quantitative 3-dimensional parameters describing the fat structure were calculated. With regard to the microstructural and rheological relationship, results from the correlation carried out show that a correlation exists among some microstructural and rheological parameters of the yogurt samples. The results from this study also show that $\mu \mathrm{CT}$ is a suitable technique for the microstructural analysis of fat as it not only quantifies the fat deposits present, but also determines the deposits' spatial distribution.
\end{abstract}

Key words: dynamic-mechanical property, fat, microstructure, x-ray microtomography, yogurt

\section{INTRODUCTION}

The microstructure of a product is particularly important for the growing number of semisolid foods and influences to a large extent the physical, textural, and sensory properties of these products, ranging from sauces and dressings to yogurt, spreads, and ice cream. Developing a proper understanding of the microstructure, particularly the spatial distribution and interaction of food components, is a key tool in developing food emulsions with desired textural and organoleptic properties. Yogurt has been produced and eaten for years, and today, it is a common food item throughout the world. It is a nutritious food with unique health

Received August 2, 2010.

Accepted October 21, 2010.

${ }^{1}$ Corresponding author: ma.delnobile@unifg.it benefits (i.e., it is rich in protein, calcium, riboflavin, vitamin $\mathrm{B}_{6}$, and vitamin $\mathrm{B}_{12}$ ). In recent years, the nutritional trend toward low-calorie foods, such as low-fat yogurts, has increased the interest in fat substitutes that do not alter the consistency or texture of the product. From this point of view, rheological and microstructural properties may provide a quantitative contribution to texture characterization and control when using different formulations (Liu et al., 2006).

With respect to semisolid food products, the microstructural quality often is assessed by sensory panels or by simple physical measurements, such as texture firmness or water exudation. Information about the $3-\mathrm{D}$ structure and ingredients of semisolid foods can now be obtained using various imaging techniques. Although a wide variety of imaging techniques exist, they are mostly invasive as they require sample preparation; hence, formation of artifacts often are restricted to certain types of food products. X-ray microtomography, on the other hand, is a noninvasive technique that has several advantages over other methods, including the ability to image low-moisture materials. Microtomography $(\boldsymbol{\mu C T})$ uses the differences in x-ray attenuation arising, principally, from differences in density within the specimen. A series of 2-dimensional x-ray images are obtained as a sample is rotated. The series of slices, covering the entire sample, can be rendered into a 3-D image that can either be presented as a whole or as virtual slices of the sample at different depths and in different directions. Manipulation and analyses of $\mu \mathrm{CT}$ data using special software also allows reconstruction of cross sections at depth increments as low as $15 \mu \mathrm{m}$, and along any desired orientation of the plane of cut. A series of noninvasive $\mu \mathrm{CT}$ slices of the same sample in any direction can provide much more information than just 1 scanning electron microscopy or optical imaging picture, for example.

Given the success of $\mu \mathrm{CT}$ in medical applications, material science, chemical engineering, geology, and biology, much attention in recent years has been focused on extending this imaging technique to food science to aid in the study of food microstructure. Microtomogra- 
phy has been used successfully to observe the stability of gas bubbles in dough during the bread-making process (Whitworth and Alava, 1999) and the microstructure of foams (Lim and Barigou, 2004), to provide a 3-dimensional quantitative analysis of breadcrumbs (Falcone et al., 2005), and to study the porous structure of bread (Falcone et al., 2004) and ice crystals within frozen foods (Mousavi et al., 2005). Recently, this technique also has been used to study the bubble size distribution in wheat flour dough (Bellido et al., 2006), the effect of far-infrared radiation-assisted drying on the microstructure of banana slices (Léonard et al., 2008), 3 -dimensional pore space quantification of apple tissue (Mendoza et al., 2007), and the role of sugar and fat in sugar-snap cookies (Pareyt et al., 2009).

Structure-property relationships can strongly affect the physiochemical, functional, technological, and even nutritional properties of foods. For example, with regard to food emulsions like ice cream, yogurt, and spreads, the consumer appreciation of these products is strongly linked to the texture. For texture, sensory properties of food emulsions are related to both mechanical properties and cellular structure. In this context, determining the relationships between a given mechanical property and the cellular structure is thus of prime importance. Processes are no longer designed from a macroscopic level; knowing the properties of foods on the micro scale determines the process specification. Hence, x-ray $\mu \mathrm{CT}$ is fast becoming a useful tool to aid in the study of food microstructure.

The aim of this study was to demonstrate the capability of x-ray microtomography as a useful tool for the quantification of fat deposits and fat distribution in food emulsion products such as yogurts; furthermore, the correlation between rheology and the microstructure of different yogurts also is investigated.

\section{MATERIALS AND METHODS}

\section{Raw Materials}

Four different types of commercially produced yogurts and 2 homemade yogurts, chosen for their variability of fat contents, were used for this experiment: Activia (Danone SpA, Milan, Italy), Muller bianco (Muller Italia, Verona, Italy), Activia 0.1\%, Muller $0.1 \%$, Vasetto B, and Vasetto A. The homemade yogurts, Vasetto A and Vasetto B were produced in our laboratory, whereas the commercially produced yogurts were purchased locally. All tests, microstructural and rheological, were carried out on the same day, $310-\mathrm{mL}$ samples were prepared for each type of yogurt; with regard to $\mu \mathrm{CT}$ analysis, each sample was placed in a cylindrical tube.

\section{Preparation of the Homemade Yogurts}

One liter of skimmed UHT milk was treated in an autoclave at $90^{\circ} \mathrm{C}$ for $5 \mathrm{~min}$ and then cooled to approximately $40^{\circ} \mathrm{C}$. The heat treatment was carried out to improve the development of the inoculated microbial flora, subsequently making the clot more compact and reducing the phenomenon of serum separation. The inoculum consisted of a mixture of Lactobacillus bulgaricus and Streptococcus thermophilus (D Jointech, Centro Sperimentale del Latte, Lodi, Italy) and probiotic microorganisms Lactobacillus acidophilus LY0002 (Probiotical, Novara, Italy; Vasetto A) and Bifidobacterium lactis LY0018 (Probiotical, Italy; Vasetto B) added separately. The $0.15 \%$ inoculum was added to milk, after which the inoculated milk was mixed, portioned into the jars, and incubated in a yogurt maker (Girmi, Brescia, Italy) for 3 to $6 \mathrm{~h}$ depending on the nature of the inoculum and temperature. The incubation was stopped at a pH of 4.4 and, after incubation, the yogurt was immediately cooled to stop bacterial growth and stored at $4^{\circ} \mathrm{C}$.

\section{Tomographic Analysis}

For $\mu \mathrm{CT}$, the samples were imaged under the same conditions, using the Skyscan 1172 high-resolution desktop x-ray microtomography system (SkyScan, Kontich, Belgium). The yogurt sample was placed in a cylindrical tube; the source and the detector were fixed, and the sample was rotated during measurement. Power settings of $100 \mathrm{kVp}$ and $100 \mu \mathrm{A}$ were used. A charge couple device camera with $2,000 \times$ 1,048 pixels was used to record the transmission of the conical x-ray beam through all samples. The distance source-object-camera was adjusted to produce images with a pixel size of $2 \mu \mathrm{m}$. Four-frame averaging, a rotation step of $0.40^{\circ}$ and an exposure time of $1767 \mathrm{~ms}$ were chosen to minimize the noise, covering a view of $180^{\circ}$. Smoothing and beam-hardening correction steps were applied to suppress noise and beam-hardening artifacts, respectively. Beam-hardening correction was only moderately applied, set to $25 \%$ within NRecon (SkyScan), and a fast ring artifacts reduction (set to 51 within NRecon) also was applied. Once initial parameters were set, the acquisition step was completely automated and did not require operator assistance. Scan time, on average, required $30 \mathrm{~min}$. A set of flat cross-section images was obtained for each sample after tomographical reconstruction by the reconstruction software NRecon. Three-dimensional reconstructions of samples were created by stacking all 2-dimensional tomographs, a total of 125 slice images with a slice spacing of $0.069 \mathrm{~mm}$. 
For image processing and analysis, the SkyScan software, CT-Analyzer (CTAn), was used. Image segmentation was first carried out on the smoothed 8-bit, gray-scale images obtained from the reconstruction step using CTAn software. Segmentation is the process of converting the gray-scale images into black-and-white images by assigning the value 1 to all pixels whose intensity was below a given gray-tone value and 0 to all the others. For this, an automatic threshold based on the entropy of the histogram (Sahoo et al., 1997) was calculated for each image. The lower gray threshold (8) and upper gray threshold (110) values were identified; each sample was processed under the same conditions.

For data analysis, before 3-dimensional reconstruction, a component-labeling algorithm, available within CTAn, was used to isolate the largest 3-dimensional connected structures. All reconstructions were created in CTAn using an adaptive rendering (locality 10 and tolerance 0.25) algorithm and saved as a P3G surface model (SkyScan model format). The P3G models were then imported into CT vol software (SkyScan) for visualization.

The following 7 geometric parameters were measured using the CTAn software: percent object volume (POV), object surface:volume ratio (OSVR), fragmentation index (FI), structure thickness (ST), structure separation (SS), structure modeling index (SMI), and degree of anisotropy (DA), where (1) POV is the proportion of the volume of interest occupied by binarized solid objects (i.e., fat); (2) OSVR is the basic parameter in characterizing the complexity of the structures and also is the basis of model-dependent estimates of thickness (i.e., size and distribution of fat present in each sample); (3) FI is an index of connectivity of structure, which was developed and defined by Hahn et al., (1992); it calculates the index of relative convexity or concavity of the total surface of the sample; (4) ST calculates or estimates the true structure thickness of the sample from 2-dimensional measurements; (5) SS is essentially the thickness of the spaces as defined by binarization within the volume of interest (i.e., fat); it also can be calculated either from 2-dimensional images with model assumptions or directly in 3 dimensions; (6) SMI estimates the characteristic form of which the structure is composed (i.e., whether it is more plate-like, rod-like or even sphere-like; $0=$ ideal plate, $3=$ cylinder, and $4=$ sphere); and (7) DA, which is a measure of preferential alignment of fat.

\section{Rheological Analyses}

The dynamic-mechanical properties of the 4 yogurt samples were studied using a controlled-strain rotational rheometer (ARES model, TA Instruments, New Castle,
DE) equipped with a force rebalance transducer (model 1K-FRTN1, 1-1,000 gcm, $200 \mathrm{rad} / \mathrm{s}, 2-2,000$ grams force) and parallel plates (superior plate diameter of 50 $\mathrm{mm}$ ). A steady temperature was ensured with an accuracy of $\pm 0.1^{\circ} \mathrm{C}$ by means of a controlled fluid bath unit and an external thermostatic bath. To prevent water evaporation, a suitable cover tool sealing the top of the superior plate was used during testing. Storage modulus $\left(\mathbf{G}^{\prime}\right)$ and loss modulus $\left(\mathrm{G}^{\prime \prime}\right)$ were determined in a frequency range of 0.01 to $10 \mathrm{~Hz}$. The strain value was obtained by preliminary strain sweep oscillatory trials to determine the linear viscoelastic region. The strain sweep oscillatory tests were carried out at a frequency of $1 \mathrm{~Hz}$ and in a range of shear strain of 0.01 to $99 \%$. All experiments were carried out at $4^{\circ} \mathrm{C}$. Three repetitions of the dynamic mechanical experiments were performed for each yogurt sample.

\section{Chemical Analyses}

The yogurt samples were analyzed for protein by the Kjeldahl method according to the AOAC (1995) method, and for fat by the Gerber method according to the British Standards Institution (1989).

\section{Statistical Analysis}

Data were subjected to ANOVA using the SPSS statistical software (Somers, NY). The mathematical model for $\mu \mathrm{CT}$ parameters and rheological parameters included fixed effect. When significant effects were found (at $P<0.05$ ), Student's $t$-test was used to locate significant differences between means. Pearson correlation coefficients (CORR procedure) were calculated to examine the relationship between the rheological parameters and all $\mu \mathrm{CT}$ parameters.

\section{RESULTS AND DISCUSSION}

To assess the validity of $\mu \mathrm{CT}$, the chemical analysis was performed on the yogurt samples. Furthermore, the additional information gained from the $\mu \mathrm{CT}$ analysis (i.e., the geometric parameters mentioned above) provided the required information to characterize and investigate the correlations, if any, among the microstructures and the rheological properties of the yogurt samples.

\section{Microtomography Technique Validation}

With regard to $\mu \mathrm{CT}$ analysis, Figures 1a to $1 \mathrm{f}$ show the gray-level reconstructed cross-section images of the samples acquired by $\mu \mathrm{CT}$; the contrasts in these images are based on the differences in absorption of x-rays by 


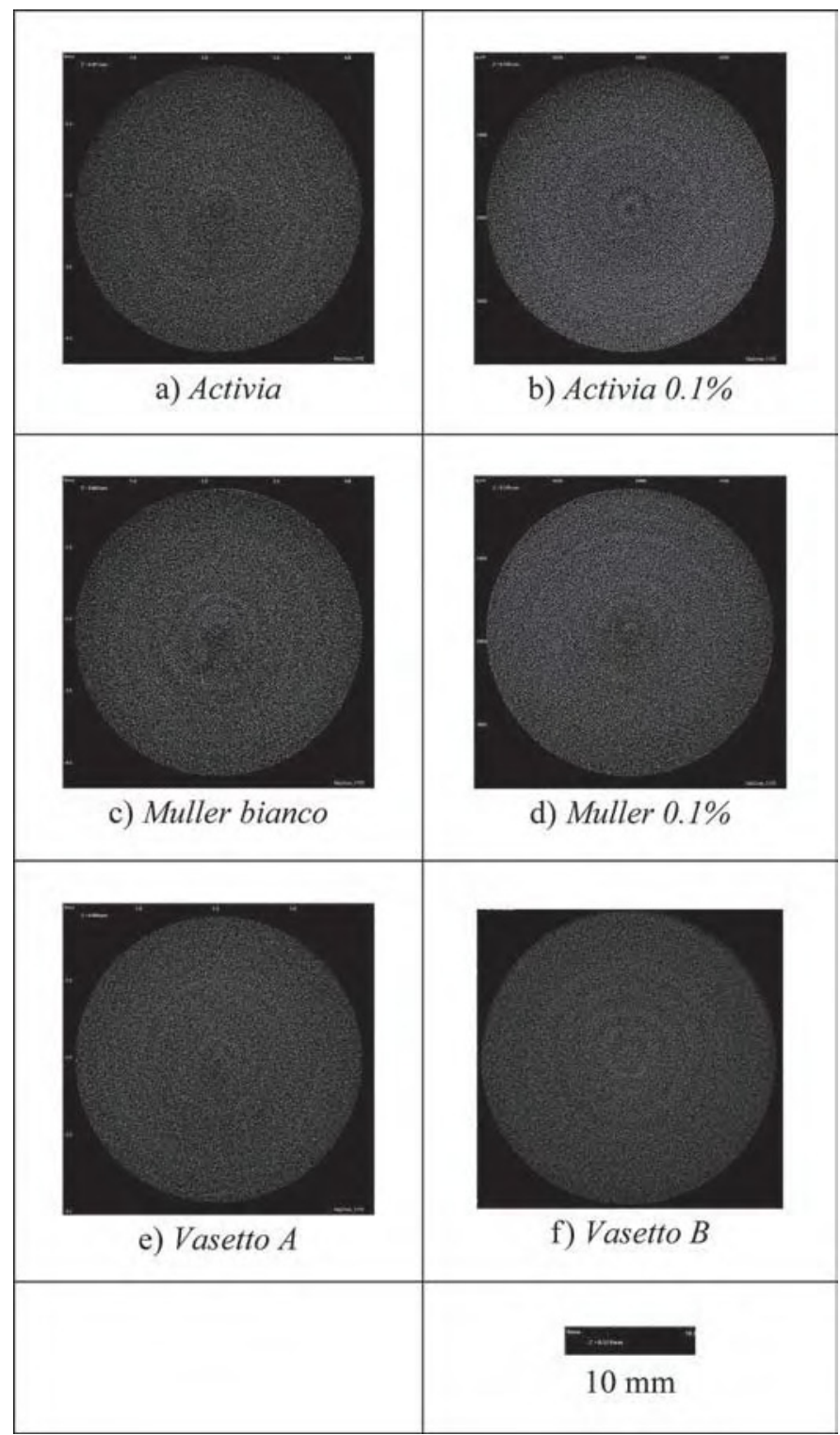

Figure 1. Grayscale tomography of the yogurt samples Activia (Danone SpA, Milan, Italy), Muller (Muller Italia, Verona, Italy), and Vasetto (produced in our laboratory).

the constituents of the sample (e.g., fat and protein). This contrast is produced by a variation of density and a change in composition of the sample and is based exclusively on the detection of an amplitude variation of x-rays transmitted through the sample itself. The obtained image is a map of the spatial distribution of the $\mathrm{x}$-rays in which the brighter regions correspond to the higher level of attenuation (i.e., higher density region). It can be assumed from the figures (Figure 1a to 1f) that the darkest gray areas represent fat as it has a lower absorption coefficient with respect to protein. Further information cannot be acquired from these im-

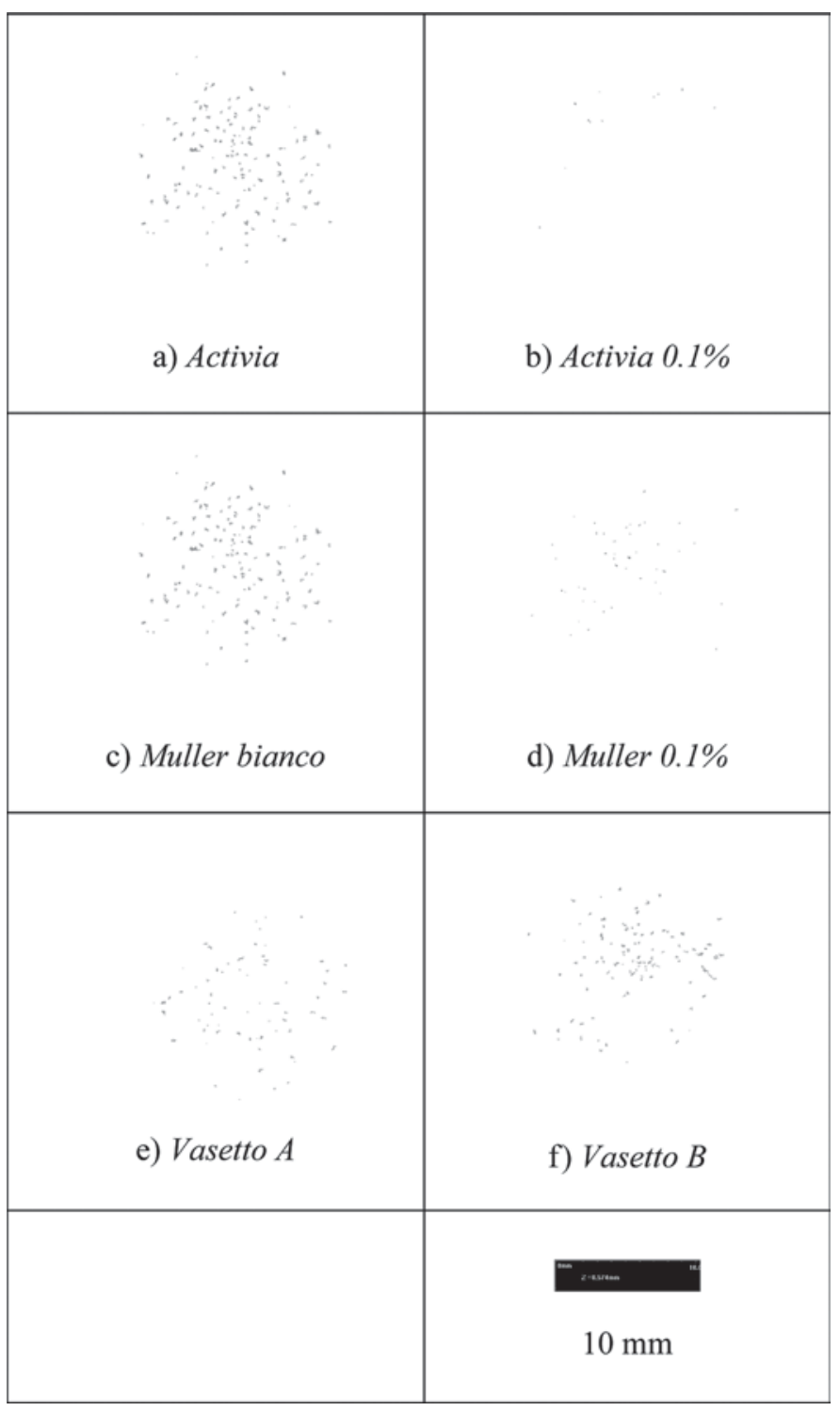

Figure 2. Three dimensional reconstruction of the yogurt samples using microtomography. Yogurt samples were from Activia (Danone SpA, Milan, Italy), Muller (Muller Italia, Verona, Italy), or were produced in our laboratory (Vasetto).

ages, as these are only 2-dimensional representations of the samples. On the other hand, Figures $2 \mathrm{a}$ to $2 \mathrm{f}$ show the 3-dimensional reconstructions for all the samples from which the geometrical parameters were calculated using the CTAn software. The percentage fat volume (i.e., the geometric parameter POV) was calculated for each image as a representation of the percentage of total fat content within the sample.

Table 1 shows the values for the fat content determined by chemical analysis and the average POV values of the yogurt samples obtained by $\mu \mathrm{CT}$ analysis. It can be noted from this table that no statistically significant 
Table 1. Percentage of fat measured by x-ray microtomography technique and the fat content measured by chemical analysis (per 100 $\mathrm{mL}$ of product)

\begin{tabular}{lcc}
\hline Yogurt $^{1}$ & $\begin{array}{c}\text { Average object } \\
\text { Volume }(\%)\end{array}$ & $\begin{array}{c}\text { Average fat } \\
\text { content }\end{array}$ \\
\hline Activia & $4.49^{\mathrm{a}}$ & $4.55^{\mathrm{a}}$ \\
Muller bianco & $5.52^{\mathrm{b}}$ & $5.6^{\mathrm{b}}$ \\
Vasetto A & $2.67^{\mathrm{c}}$ & $2.75^{\mathrm{c}}$ \\
Vasetto B & $2.23^{\mathrm{d}}$ & $2.20^{\mathrm{d}}$ \\
Activia 0.1\% & $0.92^{\mathrm{e}}$ & $1.00^{\mathrm{e}}$ \\
Muller 0.1\% & $0.71^{\mathrm{f}}$ & $0.65^{\mathrm{f}}$ \\
\hline
\end{tabular}

${ }^{\mathrm{a}-\mathrm{f}}$ Mean in the same column followed by different superscript letters differ significantly $(P<0.05)$.

${ }^{1}$ Activia is from Danone SpA, Milan, Italy; Muller is from Muller Italia, Verona, Italy; Vasetto is produced in our laboratory.

differences existed among the POV values and the fat content values as determined by chemical analysis. This high correlation between the 2 methods for the determination of fat content highlights the validity of $\mu \mathrm{CT}$; therefore, this analyzing technique produces an accurate quantification of the fat content present in yogurt.

\section{Application of $\mu C T$ Technique}

Table 2 shows the average values obtained for the following 7 parameters using the CTAn software-POV, OSVR, FI, ST, SS, SMI, and DA - as well as the results of the statistical analysis carried out as reported above. The FI is the index of connectivity and is a measure of relative convexity or concavity of the total solid surface, based on the principle that concavity indicates connectivity, and convexity indicates isolated disconnected structures (Lim and Barigou, 2004). A lower FI signifies better-connected solid lattices and has a negative index; a higher FI indicates a more disconnected solid structure and has a positive index. As can be noted from the results, the FI is positive for all samples and no statistically significant differences were found among the samples; therefore, the fat structures in these samples have disconnected solid lattices and are convex in structure. The DA is a measure of the 3 -dimensional structural symmetry (i.e., in this case, it indicates the presence or absence of preferential alignment of the fat present along a particular direction; Lim and Barigou, 2004). A value of 0 would correspond to total isotropy, whereas a value of 1 would indicate total anisotropy. According to the results obtained for DA (see Table 2), the fat present in all samples are isotropic even though statistical differences did exist among the samples; this in accordance with Langton et al. (1998), who found out that the fat droplets in emulsion food samples had no orientation and could be regarded as isotropic.

The Muller bianco and Activia samples have the highest POV values, but this is because these 2 are not considered "low-fat" yogurts. The low-fat yogurts, Activia $0.1 \%$ and Muller $0.1 \%$, have only a trace of fat present. The homemade yogurts, Vasetto A and Vasetto $\mathrm{B}$, have higher fat contents compared with the low-fat yogurts, but they have much less fat content than the other 2 commercially produced yogurt samples, Muller bianco and Activia. The OSVR indicates the fat droplet distribution within the sample. The higher the value, the more finely distributed the fat droplets present in the sample. It can be observed from the table that the Activia $0.1 \%$ and Muller $0.1 \%$ samples have the highest OSVR values that are also statistically equal; therefore, they have a much finer dispersion of fat globules compared with the other 2 samples. On the other hand, the Muller bianco and Activia samples have the lowest OSVR values and also were statistically equal (i.e., they had approximately the same type of fat distribution). Their low values indicate that the fat droplets present in these samples are more largely distributed with respect to the other 2 samples. The ST is the average

Table 2. The average values of the geometric parameters for the yogurt samples ${ }^{1}$

\begin{tabular}{|c|c|c|c|c|c|c|c|}
\hline Yogurt $^{2}$ & $\begin{array}{c}\text { Object } \\
\text { volume (\%) }\end{array}$ & $\begin{array}{c}\text { Object } \\
\text { surface:volume } \\
\text { ratio }(1 / \mu \mathrm{m})\end{array}$ & $\begin{array}{l}\text { Fragmentation } \\
\text { index }(1 / \mu \mathrm{m})\end{array}$ & $\begin{array}{c}\text { Structure } \\
\text { modeling } \\
\text { index }^{3}\end{array}$ & $\begin{array}{l}\text { Structure } \\
\text { thickness } \\
(\mu \mathrm{m})\end{array}$ & $\begin{array}{c}\text { Structure } \\
\text { separation } \\
(\mu \mathrm{m})\end{array}$ & $\begin{array}{c}\text { Degree of } \\
\text { anisotropy }^{3}\end{array}$ \\
\hline Activia & $4.49^{\mathrm{a}}$ & $0.48^{\mathrm{a}}$ & $0.14^{\mathrm{a}}$ & $2.43^{\mathrm{a}}$ & $2.21^{\mathrm{a}}$ & $54.78^{\mathrm{a}}$ & $0.35^{\mathrm{a}}$ \\
\hline Muller bianco & $5.52^{\mathrm{a}}$ & $0.51^{\mathrm{a}}$ & $0.12^{\mathrm{a}}$ & $2.41^{\mathrm{a}}$ & $2.11^{\mathrm{a}}$ & $55.13^{\mathrm{a}}$ & $0.24^{\mathrm{b}}$ \\
\hline Vasetto A & $2.67^{\mathrm{b}}$ & $1.39^{\mathrm{b}}$ & $0.13^{\mathrm{a}}$ & $1.67^{\mathrm{b}}$ & $6.97^{\mathrm{b}}$ & $33.20^{\mathrm{b}}$ & $0.32^{\mathrm{a}}$ \\
\hline Vasetto B & $2.23^{\mathrm{b}}$ & $1.20^{\mathrm{c}}$ & $0.12^{\mathrm{a}}$ & $1.75^{\mathrm{b}}$ & $7.74^{\mathrm{c}}$ & $34.56^{\mathrm{b}}$ & $0.20^{\mathrm{b}}$ \\
\hline Activia $0.1 \%$ & $0.92^{\mathrm{c}}$ & $1.85^{\mathrm{d}}$ & $0.14^{\mathrm{a}}$ & $2.76^{\mathrm{a}}$ & $2.10^{\mathrm{a}}$ & $170.00^{\mathrm{c}}$ & $0.29^{\mathrm{a}}$ \\
\hline Muller $0.1 \%$ & $0.71^{\mathrm{c}}$ & $1.76^{\mathrm{d}}$ & $0.13^{\mathrm{a}}$ & $2.56^{\mathrm{a}}$ & $2.20^{\mathrm{a}}$ & $169.23^{\mathrm{c}}$ & $0.32^{\mathrm{a}}$ \\
\hline
\end{tabular}

${ }^{\mathrm{a}-\mathrm{d}}$ Mean in the same column followed by different superscript letters differ significantly $(P<0.05)$.

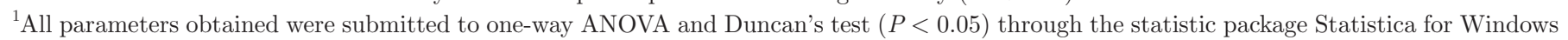
(Statsoft, Tulsa, OK).

${ }^{2}$ Activia is from Danone SpA, Milan, Italy; Muller is from Muller Italia, Verona, Italy; Vasetto is produced in our laboratory.

${ }^{3}$ Adimensional parameter. 


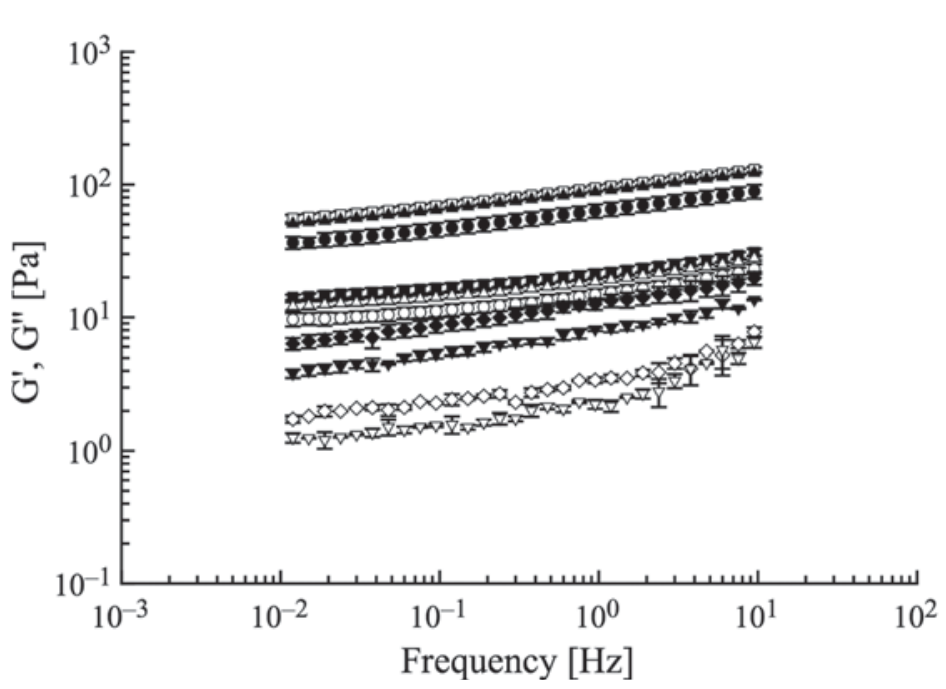

- $\quad$ ' muller bianco

- G' muller bianco 0.1

- $\quad$ G' Activia naturale 0.1

- G" muller bianco

$\triangle \quad$ G" muller bianco 0.1

- $\quad$ G" Activia naturale 0.1

- $G^{\prime} A$

- G'B

$\diamond \quad G " A$

$\nabla \quad$ G" B

Figure 3. Illustration of the storage modulus $\left(\mathrm{G}^{\prime}\right)$ and loss modulus $\left(\mathrm{G}^{\prime \prime}\right)$ values versus the oscillatory frequency for the yogurt samples. $\mathrm{A}=$ Vasetto $\mathrm{A} ; \mathrm{B}=$ Vasetto $\mathrm{B}$. The order of yogurt samples in the legend (from top to bottom) corresponds to the order of the curves at the lowest oscillatory frequency.

thickness of the fat structure present; this parameter calculates a volume-based thickness of the structure in 3 dimensions independent of an assumed structure type. It can be noted from Table 2 that the Vasetto $\mathrm{A}$ and Vasetto B samples have the highest ST values; therefore, they have a similar fat thickness, whereas the commercially produced yogurts have lower ST values that are statistically equal. The SS is the average distance between the fat structures in the samples; the homemade yogurts Vasetto A and Vasetto B, and the commercially produced yogurts Muller bianco and Activia have low SS values. The Activia $0.1 \%$ and Muller $0.1 \%$ light samples have high SS values. This could be because the fat content in these samples is very low and the average distance between each fat globule is greater with respect to the other samples.

The SMI values calculated for all the samples ranged from 1.67 to 2.76 , and significant differences existed among the SMI index values for the commercially produced yogurts and the homemade yogurt samples. The SMI parameter, a topological index, gives an estimate of the characteristic shape of a structure in terms of plates and cylinders composing the 3-dimensional structure (Hildebrand and Rüegsegger, 1997) and is calculated using a differential analysis of the triangulated surface of the structure under examination. As stated above, the SMI assumes integer values of 0,3 , and 4 for ideal plates, cylinder, and spheres, respectively. Due to the lack of literature on the shape of the fat droplets in yogurts, these results cannot be further analyzed; therefore, further investigation will be carried out to understand the above SMI results.

\section{Rheological Analysis}

Figure 3 shows the rheological data, $\mathrm{G}^{\prime}$ and $\mathrm{G}^{\prime \prime}$ values vs. the oscillatory frequency for the yogurt samples. It can be inferred from this figure that all samples exhibited viscoelastic behavior with $\mathrm{G}^{\prime}$ greater than the loss modulus (G") (Rao and Steffe, 1992; Subramanian and Gunasekaran, 1997) and that all the yogurt samples exhibited solid-like gel behavior with rheological spectra resembling that of weak gel (Ross-Murphy, 1988; Richardson et al., 1989). Typical weak gel characteristics were observed: $G^{\prime}$ was greater than $G^{\prime \prime}$ throughout the frequency range, and the moduli showed a slight dependence on frequency.

The experimental data on the frequency sweep tests were correlated using the following power law according to Bohlin's theory of flow as a cooperative phenomenon (Bohlin, 1980):

$$
\mathrm{G}^{\prime}=\mathrm{A} \cdot \omega \cdot \exp (1 / \mathrm{z}),
$$

where $\omega$ is the frequency. On the basis of this theory, emulsions are modeled as a network of rheological units that interact to establish system structure. The coordination number $\mathrm{z}$ gives the level of these interactions and the coefficient $\mathrm{A}$ their magnitude. The $\mathrm{z}$ and $\mathrm{A}$ values were obtained for all samples and are shown in Table 3. These parameters give an idea of the emulsion stability. It can be seen that the all the commercially produced yogurt samples with respect to the homemade yogurts had the highest $\mathrm{z}$ values that were also statistically equal. Therefore, the commercially produced yogurt 
Table 3. The coordination number $\mathrm{z}$ and the coefficient A values for the yogurt samples

\begin{tabular}{lrlcl}
\hline Yogurt & $\mathrm{A}$ & \multicolumn{1}{c}{$\mathrm{dA}^{1}$} & $\mathrm{z}$ & $\mathrm{dz}^{1}$ \\
\hline Activia & 75.65 & 0.17 & 7.42 & $6.7 \mathrm{e}-2$ \\
Muller bianco & 95.45 & 0.18 & 7.52 & $6.0 \mathrm{e}-2$ \\
Vasetto A & 12.85 & $5.04 \mathrm{e}-2$ & 5.91 & $7.7 \mathrm{e}-2$ \\
Vasetto B & 8.18 & $7.4 \mathrm{e}-2$ & 5.26 & 0.145 \\
Activia 0.1\% & 64.23 & 0.14 & 7.19 & $6.3 \mathrm{e}-2$ \\
Muller 0.1\% & 89.13 & 0.15 & 7.36 & $4.9 \mathrm{e}-2$ \\
\hline
\end{tabular}

${ }^{1} \mathrm{dA}$ and $\mathrm{dz}$ are standard deviations.

samples seem to have a more complex structure and contain more microstructural interactions with respect to the homemade yogurt samples. Regarding the A parameter, all samples differed significantly, although the homemade yogurts had the lowest values; the homemade yogurt samples were less stable with respect to the commercially produced samples.

\section{Correlation}

Table 4 shows the results of the correlation among the microstructural parameters and rheological properties of the yogurt samples. It can be noted from this table that a correlation exists among some of the microstructural and rheological parameters of the yogurt samples. With regard to the microstructural parameter correlation, a strong correlation was found among the following pairs of microstructural parameters: POV and OSVR, ST and SMI. The correlation between POV and OSVR was in coherence with Tamime and Marshall (1997), who found that for emulsions, fat content was related to droplet size and distribution.

Among the microstructural and rheological parameters, a strong correlation existed among the coordination number $\mathrm{z}$, the coefficient A, SMI, and ST, suggesting that the number of microstructural interactions and the magnitude of these interactions depended on the average thickness and shape of the fat structure present. The results obtained suggest the existence of a link between some microstructural features and rheological properties. As stated above, structure-property relationships can strongly affect the physiochemical, functional, technological, and even nutritional properties of foods. For example, with regard to food emulsions like ice cream, yogurt, spreads, and dressings such as mayonnaise, the consumer appreciation of these products is strongly linked to the texture. Therefore, this preliminary investigation allowed us to further study the main process variables that affect the yogurt characteristics involved in consumer satisfaction to promote full product acceptability.

\section{CONCLUSIONS}

The microstructure of yogurts can be successfully analyzed using x-ray $\mu \mathrm{CT}$. The fat distribution and fat droplet size could be observed and quantified in the microtomographic images. With $\mu \mathrm{CT}$, a full highresolution, 3-dimensional image of large samples can be obtained, and image analysis of the full 3-dimensional microstructure, measuring the size, shape, networking/connectivity, and distribution of various phases is possible. These measurements represent the full 3-dimensional microstructure, which is not always possible with 2-dimensional image analysis using statistical techniques. The correlation of the $\mu \mathrm{CT}$ analysis and rheological analysis identified the microstructuralrheological structure relationships; relationships among the different microstructural parameters also were identified (i.e., the relationship between fat content to droplet size and distribution). These findings are of importance as these relationships can strongly affect the physiochemical, functional, technological, and even nutritional properties of foods.

Table 4. The correlation results for all the microstructural and rheological parameters of the yogurt samples

\begin{tabular}{lccccccccc}
\hline Item $^{1}$ & POV & OSVR & FI & SMI & ST & SS & DA & A & Z \\
\hline POV & 1 & & & & & & & & \\
OSVR & $-0.9691^{*}$ & 1 & & & & & & & \\
FI & -0.4355 & 0.4069 & 1 & & & & & & \\
SMI & -0.1347 & 0.1342 & 0.4252 & 1 & & & & \\
ST & -0.0267 & 0.0129 & -0.4943 & $-0.9371^{*}$ & 1 & & & \\
SS & -0.7382 & 0.7427 & 0.6288 & 0.7157 & -0.6428 & 1 & & \\
DA & -0.0417 & 0.0316 & 0.4946 & 0.305 & -0.3702 & 0.1851 & 1 & & \\
A & 0.2318 & -0.2165 & 0.1463 & $0.8563^{*}$ & $-0.9235^{*}$ & 0.4381 & 0.3013 & 1 & \\
Z & 0.194 & -0.1614 & 0.3486 & $0.8873^{*}$ & $-0.9654^{*}$ & 0.4833 & 0.4717 & $0.9622^{*}$ & 1 \\
\hline
\end{tabular}

${ }^{1} \mathrm{POV}=$ percent object volume, OSVR $=$ object surface:volume ratio, $\mathrm{FI}=$ fragmentation index, $\mathrm{ST}=$ structure thickness, SS = structure separation, $\mathrm{SMI}=$ structure modeling index, and DA = degree of anisotropy. Emulsions are modeled as a network of rheological units that interact to establish system structure. The coordination number $\mathrm{z}$ gives the level of these interactions and the coefficient A their magnitude.

*Highly, positively, and significantly correlated. 


\section{REFERENCES}

AOAC. 1995. Official Methods of Analysis. 16th ed. Association of Official Analytical Chemists, Arlington, VA.

Bellido, G. G., M. G. Scanlon, J. H. Page, and B. Hallgrimsson. 2006. The bubble size distribution in wheat flour dough. Food Res. Int. 39:1058-1066.

Bohlin, L. 1980. A theory of flow as a cooperative phenomenon. J. Colloid Interface Sci. 74:423-434.

British Standards Institution. 1989. Determination of fat content of milk and milk products (Gerber Method). BS 696. British Standards Institution, London, UK.

Falcone, P. M., A. Baiano, F. Zanini, L. Mancini, G. Tromba, D. Dreossi, and M. A. Del Nobile. 2005. Three-dimensional quantitative analysis of bread crumb by X-ray microtomohraphy. J. Food Sci. 70:265-272.

Falcone, P. M., A. Baiano, F. Zanini, L. Mancini, G. Tromba, F. Montanari, and M. A. Del Nobile. 2004. A novel approach to the study of bread porous structure: Phase-contrast X-ray micro- tomography. J. Food Sci. 69:38-43.

Hahn, M., M. Vogel, M. Pompesius-Kempa, and G. Delling. 1992. Trabecular bone pattern factor-A new parameter for simple quantification of bone microarchitecture. Bone 13:327-330.

Hildebrand, T., and P. Ruegsegger. 1997. A new method for the model independent assessment of thickness in three dimensional images. J. Microsc. 185:67-75.

Langton, M., E. Jordanssona, A. Altskara, C. Sorensenb, and A. Hermanssona. 1998. Microstructure and image analysis of mayonnaises. Food Hydrocoll. 13:113-125.

Léonard, A., S. Blacher, C. Nimmol, and S. Devahastin. 2008. Effect of far-infrared radiation assisted drying on microstructure of banana slices: An illustrative use of X-ray microtomography in microstructural evaluation of a food product. J. Food Eng. 85:154-162.

Lim, K. S., and M. Barigou. 2004. X-ray micro-tomography of cellular food products. Food Res. Int. 37:1001-1012.

Liu, H., X. M. Xu, and D. Guo Sh. 2006. Rheological, texture and sensory properties of low-fat mayonnaise with different fat mimetics. LWT Food Sci. Technol. 40:946-954.
Mendoza, F., P. Verboven, H. M. Mebatsion, and G. Kerckhofs. 2007. Three-dimensional pore space quantification of apple tissue using X-ray computed microtomography. Planta 226:559-570.

Mousavi, R., T. Miri, P. W. Cox, and P. J. Fryer. 2005. A novel technique for ice crystal visualization in frozen solids using X-ray micro-computed tomography. J. Food Sci. 70:437-442.

Pareyt, B., F. Talhaoui, G. Kerckhofs, K. Brijs, H. Goesaert, M. Wevers, and J. A. Delcour. 2009. The role of sugar and fat in sugar-snap cookies: Structural and textural properties. J. Food Eng. 90:400-408.

Rao, M. A., and J. F. Steffe. 1992. Viscoelastic Properties of Foods. Elsevier Applied Science, New York, NY.

Richardson, R. K., E. R. Morris, S. B. Ross-Murphy, L. J. Taylor, and I. C. M. Dea. 1989. Characterisation of the perceived texture of the thickened systems by dynamic viscosity measurements. Food Hydrocoll. 3:175-191.

Ross-Murphy, S. B. 1988. Small deformation measurements. Pages 387-400 in Food Structure: Its Creation and Evaluation. J. M. V. Blanshard, and J. R. Mitchell, ed. Butterworths, London, UK.

Sahoo, P. K., T. W. Saalf, and T. A. Albert. 1997. Threshold selection using a minimal histogram entropy difference. Opt. Eng. 36:1976-1981.

Subramanian, R., and S. Gunasekaran. 1997. Small amplitude oscillatory shear studies on Mozzarella cheese. Part I. Region of linear viscoelasticity. J. Texture Stud. 28:633-642.

Tamime, A. Y., and V. M. E. Marshall. 1997 Microbiology and technology of fermented milks. Pages 57-152 in Microbiology and Biochemistry of Cheese and Fermented Milk. 2nd ed. B. A. Law, ed. Blackie Academic and Professional, London, UK.

Whitworth, M. B., and J. M. Alava. 1999. The imaging and measurement of bubble in bread doughs. Pages 221-231 in Bubbles in Foods. G. M. Campbell, C. Webb, S. S. Pandiella, and K. Niranjan, ed. Eagan Press, St. Paul, MN. 